\title{
REPRESENTATIONS OF CHEVALLEY GROUPS ARISING FROM ADMISSIBLE LATTICES
}

\author{
ZONGZHU LIN
}

(Communicated by Warren J. Wong)

\begin{abstract}
The modules for a Chevalley group arising from admissible lattices in an irreducible module for the associated complex semisimple Lie algebra are studied. It is proved that the transpose of such a module is still in this collection and generically the cohomology modules of line bundles on the flag varieties are in this collection also. In the rank 1 case, all modules in this collection are indecomposable and we hope this is true in general.
\end{abstract}

\section{INTRODUCTION}

The purpose of this paper is to investigate the modules for a Chevalley group obtained by reduction from admissible lattices in an irreducible module for the associated complex Lie algebra $\mathfrak{g}$. These modules have been studied by W. J. Wong in [9]. It is well known that different admissible lattices in a module can give rise to different modules for the Chevalley group, though they have the same character. It is still not clear what kind of modules can be obtained in this way. The Weyl modules and the induced modules are obtained from the minimal and maximal admissible lattices respectively. The structure of these modules is still a mystery.

In $\S 1$ we show that whenever $M$ is a module arising from an admissible lattice, so is its transpose $M^{\mathrm{tr}}[2,2.1]$. This is based on the contravariant form defined by W. J. Wong in [9]. Section 2 is based on the work of Andersen on the induction theory for Chevalley groups over the integers [1]. It is proved that whenever $H_{k}^{l(w)}(w \cdot \lambda)$ is the only nonvanishing cohomology group of the line bundle, it can be obtained from an admissible lattice in $V(\lambda)$. Besides these cohomology modules, there are still many other modules arising from admissible lattices. It turns out that every quotient of a Weyl module is a submodule of such a module. In order to study these modules in general, we deal with $\mathfrak{s l}_{2}$ in $\S 3$. In this case, we prove that all modules arising from admissible lattices in an irreducible module for $\mathfrak{s l}_{2}$ are indecomposable for $\mathrm{SL}_{2}$.

Communications with James E. Humphreys on this matter were very help-

Received by the editors September 28, 1990; the contents of this paper have been presented to AMS meeting \#860, October 20-21, 1990.

1980 Mathematics Subject Classification (1985 Revision). Primary 20G05. 
ful. The calculation of the $p$-adic valuations of binomial coefficients in 3.4 is motivated from a conversation with Neal Koblitz.

\section{The transpose of a module}

1.1. Let $\mathfrak{g}$ be a complex semisimple Lie algebra with root system $R$. Let $R^{+}$ be the set of positive roots and $S$ the set of simple roots. Let $W$ be the Weyl group that is generated by the simple reflections $s_{\alpha}(\alpha \in S)$ on $X \otimes \mathbf{R}$. Here $X$ is the lattice of integral weights. Throughout this paper, the tensor product is taken over $\mathbf{Z}$.

For each $\lambda \in X_{+}$(the set of dominant weights), $V(\lambda)$ denotes the simple finite dimensional $\mathfrak{g}$-module with highest weight $\lambda$. Let $U_{\mathbf{Z}}(\mathfrak{g})$ be the Kostant $\mathbf{Z}$-form in the enveloping algebra $U(\mathfrak{g})$. A Z-lattice (the Z-span of a basis) in $V(\lambda)$ is called admissible if it is $U_{\mathbf{Z}}(\mathfrak{g})$-invariant. Each admissible lattice $V$ is a direct sum of its weight spaces and we use $V_{\mu}$ to denote the weight space of weight $\mu$ in $V$. Let us fix a highest weight vector $v^{+}$in $V(\lambda)$. Then any admissible lattice in $V(\lambda)$ is proportional to an admissible lattice $V$ with $V_{\lambda}=\mathbf{Z} v^{+}$. So in this paper an admissible lattice means such a lattice with $v^{+}$ understood. It follows from [8, Lemma 19], that $\left(V_{1}\right)_{w(\lambda)}=\left(V_{2}\right)_{w(\lambda)}$ for all $w \in W$ and two admissible lattices $V_{1}$ and $V_{2}$.

Consider a Chevalley basis $\left\{x_{\alpha}, h_{i}\right\}$ in $\mathfrak{g}$. There is an involutary antiautomorphism $\tau: U(\mathfrak{g}) \rightarrow U(\mathfrak{g})$ such that $\tau\left(x_{\alpha}\right)=x_{-\alpha}$ for all $\alpha \in S[8, \mathrm{p}$. 228]. Let $\phi: V(\lambda) \rightarrow V(\lambda)_{\lambda}=\mathbf{C} v^{+} \cong \mathbf{C}$ be the projection. We define a symmetric bilinear form $\langle$,$\rangle on V(\lambda)$ by $\left\langle u_{1} v^{+}, u_{2} v^{+}\right\rangle=\phi\left(\tau\left(u_{1}\right) u_{2} v^{+}\right)$. It follows from the definition that $\left\langle V(\lambda)_{\mu}, V(\lambda)_{\nu}\right\rangle=0$ if $\mu \neq \nu .\langle$,$\rangle is non-$ degenerate and contravariant, i.e., $\left\langle u v_{1}, v_{2}\right\rangle=\left\langle v_{1}, \tau(u) v_{2}\right\rangle$ for all $u \in U(\mathfrak{g})$ and $v_{1}, v_{2} \in V(\lambda)$ (see [9]). Let $V$ be an admissible lattice in $V(\lambda)$, then $V^{\prime}=\{v \in V(\lambda) \mid\langle v, V\rangle \subseteq \mathbf{Z}\}$ is also an admissible lattice in $V(\lambda)$ with $V_{\lambda}^{\prime}=V_{\lambda}$.

Lemma. If $V_{1}$ and $V_{2}$ are two admissible lattices, then $V_{1} \subseteq V_{2}$ if and only if $V_{1}^{\prime} \supseteq V_{2}^{\prime}$. If $V_{1}^{\prime}=V_{2}$, then $V_{1}=V_{2}^{\prime}$.

It is clear that $U_{\mathbf{Z}}(\mathfrak{g}) v^{+}$is an admissible lattice and is contained in every admissible lattice. We call it the minimal admissible lattice, denoted by $V_{\min }$. By the Lemma above, $V_{\min }^{\prime}$ is a maximal admissible lattice, denoted by $V_{\max }$.

Instead of using the traditional definition of dual modules, we define the $\mathfrak{g}$ module structure on $\operatorname{Hom}_{\mathbf{C}}(M, \mathbf{C})$ by $(u f)(m)=f(\tau(u) m)$ for all $u \in U(\mathfrak{g})$, $f \in \operatorname{Hom}_{\mathbf{C}}(M, \mathbf{C})$ with $m \in M$ for each $\mathfrak{g}$-module $M$. We denote this module by $D(M)$. Let $\theta: \mathfrak{g} \rightarrow \mathfrak{g}$, with $\theta\left(x_{\alpha}\right)=-x_{-\alpha} \quad(\alpha \in S)$ and $\theta\left(h_{i}\right)=-h_{i}$, be the automorphism of $\mathfrak{g}$ as in [9, (1A)]. For each $\mathfrak{g}$-module $M, M^{\theta}$ denotes the $\mathfrak{g}$-module $M$ with the new action $x * m=\theta(x) m$. Then $D(M)=\left(M^{*}\right)^{\theta}=$ $\left(M^{\theta}\right)^{*}$. Here $*$ means the traditional dual module for $\mathfrak{g}$. We can define $D_{\mathbf{Z}}(V)$ and $D_{k}(M)$ similarly for any admissible lattice $V$ and $U_{\mathbf{Z}}(\mathfrak{g}) \otimes k$-module $M$ for any field $k$. Thus one has $D_{\mathbf{Z}}(V) \otimes k=D_{k}(V \otimes k)$ and $V^{\prime}=D_{\mathbf{Z}}(V)$ for any admissible lattice $V$.

1.2. Let $G_{\mathbf{Z}}$ be the group scheme defined by Kostant from the $\mathbf{Z}$-form $U_{\mathbf{Z}}(\mathfrak{g})$ [7]. It turns out that $U_{\mathbf{Z}}(\mathfrak{g})$ is the distribution algebra of $G_{\mathbf{Z}}$. Each admissible lattice $V \subseteq V(\lambda)$ is a rational $G_{\mathbf{Z}}$-module. Let $k$ be any field. Denote by $G_{k}$ the simply connected semisimple algebraic group defined over $k$ through base field change from $G_{\mathbf{Z}}$. Then $U_{\mathbf{Z}}(\mathfrak{g}) \otimes k$ is the distribution algebra of $G_{k}$ and $V \otimes k$ is a rational $G_{k}$-module. 
Let $\operatorname{tr}: G_{\mathbf{Z}} \rightarrow G_{\mathbf{Z}}$ be the composite of the inversion map $g \mapsto g^{-1}$ and the involutory automorphism $\phi$ in [9] $(2 \mathrm{H})$. Then $\operatorname{tr}$ is an involutory antiautomorphism and is called the transpose map by Doty and Sullivan [2, (2.1)]. For each $G_{\mathbf{Z}}$-module $M$, we define the rational $G_{\mathbf{Z}}$-module $M^{\text {tr }}$ as the $\mathrm{Z}$-module $\operatorname{Hom}_{\mathbf{Z}}(M, \mathbf{Z})$ with the action of $G_{\mathbf{Z}}$ defined through $\operatorname{tr}$ as in loc.cit. The antiautomorphism $\operatorname{tr}$ is compatible with base field change and $D_{\mathbf{Z}}(V)=V^{\tau}$ for all admissible lattices $V$.

Proposition. For each admissible lattice $V$, we have an isomorphism of $G_{k}$ modules $V^{\prime} \otimes k \cong(V \otimes k)^{\text {tr }}$.

Remark. We call the functor $M \mapsto M^{\text {tr }}$ the duality functor. The proposition shows that the collection of modules for $G_{k}$ arising from admissible lattices is invariant under the duality functor.

\section{COHOMOLOGY MODULES}

2.1. In [1], Andersen has set up the induction and the associated cohomology theory for $G_{\mathbf{Z}}$. We use the notations from his paper and let $H^{i}(\lambda)=R^{i} \operatorname{Ind}_{B_{\mathbf{Z}}}^{G_{\mathbf{Z}}}(\lambda)$ for each integral weight $\lambda \in X$. Here $B_{\mathbf{Z}}$ is the Borel subgroup of $G_{\mathbf{Z}}$ corresponding to the set of negative roots and $\lambda$ represents the rank 1 rational $B_{\mathbf{Z}}$-module. If $k$ is a field we set $H_{k}^{i}(\lambda)=R^{i} \operatorname{Ind}_{B_{k}}^{G_{k}}(\lambda)$. It follows from the universal coefficient theorem that there is an exact sequence of $G_{k}$-modules

$$
0 \rightarrow H^{i}(\lambda) \otimes k \rightarrow H_{k}^{i}(\lambda) \rightarrow \operatorname{Tor}_{1}^{Z}\left(H^{i+1}(\lambda), k\right) \rightarrow 0 .
$$

In general, $H^{i}(\lambda)$ is not free over $\mathrm{Z}$. We will denote by $H_{t}^{i}(\lambda)$ the torsion submodule and by $H_{f}^{i}(\lambda)$ the free quotient of $H^{i}(\lambda)$ by $H_{t}^{i}(\lambda)$. It follows from Andersen [1, 2.6] that if $\lambda$ is dominant, then $H_{f}^{i}(w \cdot \lambda)=0$ for $i \neq l(w)$. Furthermore, $H^{0}(\lambda)$ and $H^{N}\left(w_{0} \cdot \lambda\right)$ are free of finite rank. Here $w_{0}$ is the longest element in $W$ and $N=l\left(w_{0}\right)$.

2.2. From now on we fix a dominant weight $\lambda$. Let $m^{+} \in H^{N}\left(w_{0} \cdot \lambda\right)_{\lambda}$ be a generator of the rank 1 free $\mathbf{Z}$-module. Let $M=U_{\mathbf{Z}}(\mathfrak{g}) m^{+}$be the $G_{\mathbf{Z}^{-}}$ submodule in $H^{N}\left(w_{0} \cdot \lambda\right)$ generated by $m^{+}$. It is well known that $M \otimes k=$ $H^{N}\left(w_{0} \cdot \lambda\right) \otimes k$ is the Weyl module for $G_{k}$ for any field $k$ regardless of the characteristic. This forces $M=H^{N}\left(w_{0} \cdot \lambda\right)$.

2.3. By constructing an explicit intertwining homomorphism in the rank 1 case, in [1], Andersen gets a homomorphism of $G_{\mathrm{Z}}$-modules $T_{s_{\alpha}}: H^{l\left(s_{\alpha} w\right)}\left(s_{\alpha} w \cdot \lambda\right) \rightarrow$ $H^{l(w)}(w \cdot \lambda)$ for $w \in W$ and a simple root $\alpha$ with $l\left(s_{\alpha} w\right)=l(w)+1$ from a degenerate Grothendieck spectral sequence. $T_{s_{\alpha}}$ induces a homomorphism,

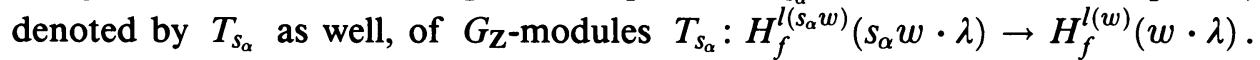
By tensoring with $\mathbf{C}$, one sees that $T_{s_{\alpha}} \otimes 1$ is an isomorphism. Therefore $T_{s_{\alpha}}$ is injective on the free part. For each $w \in W$, by considering a reduced expression of $w$ in terms of simple reflections and composing the intertwining homomorphisms constructed above, we get an embedding $H_{f}^{l(w)}(w \cdot \lambda) \rightarrow H^{0}(\lambda)$. The composite does not depend on the choice of reduced expressions of $w$ up to a multiplication by -1 . So we simply write $H_{f}^{l(w)}(w \cdot \lambda) \subseteq H^{0}(\lambda)$. In particular, we have $H^{N}\left(w_{0} \cdot \lambda\right) \subseteq H^{0}(\lambda)$.

Let us choose a generator $v_{w}$ in $H_{f}^{l(w)}(w \cdot \lambda)_{\lambda}$ for each $w \in W$. It follows from $[1,4.4]$ that $T_{s_{\alpha}}\left(v_{s_{\alpha}} w\right)= \pm v_{w} \quad\left(\right.$ if $\left.l\left(s_{\alpha} w\right)=l(w)+1\right)$. 
Since $H^{0}(\lambda) \otimes \mathbf{C}=V(\lambda)$ (Borel-Bott-Weil theorem), we can embed $H^{0}(\lambda)$ in $V(\lambda)$ and identify $v^{+}$with $v_{1} \otimes 1$. Therefore $H^{0}(\lambda)$ is an admissible lattice in $V(\lambda)$. Under the inclusion $H_{f}^{l(w)}(w \cdot \lambda) \subseteq H^{0}(\lambda)$ we can embed $H_{f}^{l(w)}(w \cdot \lambda)$ into $V(\lambda)$. They are admissible lattices in $V(\lambda)$. By considering the image of the generator $v_{w}$ in $H^{0}(\lambda)$, we get $H_{f}^{l(w)}(w \cdot \lambda)_{\lambda}=H^{0}(\lambda)_{\lambda}$. In particular $H^{N}\left(w_{0} \cdot \lambda\right)$ is the minimal admissible lattice.

Theorem. Under the embedding $H^{0}(\lambda) \subseteq V(\lambda)$, all $H_{f}^{l(w)}(w \cdot \lambda)$ are admissible lattices for $w \in W$. Furthermore, $H^{N}\left(w_{0} \cdot \lambda\right)$ is minimal and $H^{0}(\lambda)$ maximal. Proof. We only need to show $H^{0}(\lambda) \cong V_{\max }=V_{\min }^{\prime}$. But this follows from 1.2 and the Serre duality over $\mathbf{Z}[1,2.10]$ or rather its transpose form [2, 2.2].

2.4. Let us fix a prime $p$ and a field $k$ of characteristic $p$.

Corollary. Let $\lambda \in X_{+}$. If $H_{k}^{i}(w \cdot \lambda)=0$ for $i \neq l(w)$, then $H_{k}^{l(w)}(w \cdot \lambda) \cong$ $H_{f}^{l(w)}(w \cdot \lambda) \otimes k$. Thus $H_{k}^{l(w)}(w \cdot \lambda)$ arises from an admissible lattice in $V(\lambda)$.

Proof. It follows from the universal coefficient theorem, $H^{l(w)}(w \cdot \lambda) \otimes k \subseteq$ $H_{k}^{l(w)}(w \cdot \lambda)$. It is clear that $\operatorname{dim}_{k} H^{l(w)}(w \cdot \lambda) \otimes k \geq \operatorname{dim}_{k} H_{f}^{l(w)}(w \cdot \lambda) \otimes k=$ $\operatorname{dim}_{\mathbf{C}} V(\lambda)$. On the other hand, the standard vanishing property shows that $\operatorname{dim}_{k} H_{k}^{l(w)}(w \cdot \lambda)=\operatorname{dim}_{k} H_{k}^{0}(\lambda)=\operatorname{dim}_{\mathbf{C}} V(\lambda)$. By comparing the dimensions we have the isomorphism in the corollary. The second conclusion follows from 2.3.

2.5. Though there are a lot of admissible lattices in $V(\lambda)$, for a fixed field $k$, there are many admissible lattices yielding isomorphic modules for $G_{k}$. One may ask if there is any module arising from an admissible lattice and not isomorphic to any one of the cohomology modules in the generic case. If $\lambda$ is in the closure of the bottom alcove, all modules arising from admissible lattices in $V(\lambda)$ are simple with the same character and, therefore, isomorphic. In general, the following theorem says that there are many other modules arising from admissible lattices besides the cohomology modules (in the generic case). For $A_{1}$, we will see, in $\S 3$, that for $\lambda<p^{2}$, the cohomology modules are the only possible modules arising from admissible lattices.

Theorem. For $\lambda \in X_{+}$and each $G_{\mathbf{F}_{p}}$-submodule $0 \neq M \subseteq H^{0}(\lambda) \otimes \mathbf{F}_{p}$, there is an admissible lattice $V$ such that $M$ is a quotient of $V \otimes \mathbf{F}_{p}$.

Proof. Let us consider the quotient map $\sigma: H^{0}(\lambda) \rightarrow H^{0}(\lambda) \otimes \mathbf{F}_{p}$. The image of $H^{N}\left(w_{0} \cdot \lambda\right)$ is the simple socle of $H^{0}(\lambda) \otimes \mathbf{F}_{p}$. Thus the inverse image $V$ of $M$ in $H^{0}(\lambda)$ contains $H^{N}\left(w_{0} \cdot \lambda\right)$ and is, therefore, an admissible lattice. The induced map $V \otimes \mathbf{F}_{p} \rightarrow H^{0}(\lambda) \otimes \mathbf{F}_{p}$ from the inclusion has image $M$.

\section{INDECOMPOSABILITY}

3.1. In this section we assume that $\mathfrak{g}=\mathfrak{s l}_{2}$, which has a basis $\{x, y, h\}$. In this case, we write $\lambda=n \geq 0$ as a dominant weight. This paragraph contains the exercises in Humphreys' book [4] and we include them here as our starting point. $V_{\max }$ has a $\mathrm{Z}$-basis $\left\{v_{0}, v_{1}, \ldots, v_{n}\right\}$ with the following properties:

$$
h v_{i}=(n-2 i) v_{i}, \quad y v_{i}=(n-i) v_{i+1}, \quad x v_{i}=i v_{i-1} .
$$


The set $\left\{\left(\begin{array}{l}n \\ i\end{array}\right) v_{i}\right\}_{i=0}^{n}$ is a $\mathbf{Z}$-basis for $V_{\min }$.

Let $M$ be an admissible lattice with a $\mathbf{Z}$-basis $\left\{z_{0}, z_{1}, \ldots, z_{n}\right\}$. We write $z_{i}=x_{i} v_{i}$ for $i=0, \ldots, n$ for a sequence of nonzero integers $\left\{x_{0}, x_{1}, \ldots, x_{n}\right\}$. Since $M$ is an admissible lattice,

$$
\begin{aligned}
& \frac{x^{i}}{i !} z_{j}=x_{j} \frac{x^{i}}{i !} v_{j}=\frac{x_{j} j(j-i) \cdots(j-i+1)}{i ! x_{j-i}} z_{j-i}=\frac{x_{j}}{x_{j-i}}\left(\begin{array}{c}
j \\
i
\end{array}\right) z_{j-i}, \\
& \frac{y^{i}}{i !} z_{j}=x_{j} \frac{y^{i}}{i !} v_{j}=\frac{x_{j}(n-j) \cdots(n-j-i+1)}{i ! x_{j+i}} z_{j+i}=\frac{x_{j}}{x_{j+i}}\left(\begin{array}{c}
n-j \\
i
\end{array}\right) z_{j+i}
\end{aligned}
$$

are in $M$. Thus $\frac{x_{j}}{x_{j-i}}\left(\begin{array}{l}j \\ i\end{array}\right)$ and $\frac{x_{j}}{x_{j+i}}\left(\begin{array}{c}n-j \\ i\end{array}\right)$ have to be integers for all appropriate $i$ and $j$.

Lemma. Each admissible lattice $M$ in $V(n)$ is determined by a sequence of nonzero integers $\left\{x_{0}, \ldots, x_{n}\right\}$ with $x_{0}= \pm 1$ and

$$
\frac{x_{j}}{x_{i}}\left(\begin{array}{l}
j \\
i
\end{array}\right) \in \mathbf{Z} \quad \text { and } \quad \frac{x_{i}}{x_{j}}\left(\begin{array}{l}
n-i \\
n-j
\end{array}\right) \in \mathbf{Z}, \quad \text { for all } 0 \leq i<j \leq n .
$$

Proof. It follows from the above argument, for each $M$, the coefficients $\left\{x_{0}\right.$, $\left.\ldots, x_{n}\right\}$ satisfy the condition in the lemma. Conversely, if the coefficients satisfy the above condition, then $M$ is an admissible lattice.

Remark. By changing the signs of the coefficients, we can assume the $x_{i}$ 's are positive. Thus two admissible lattices are the same if and only if the associated sequences are the same.

3.2. From now on, we fix a prime $p$ and a nonnegative integer $n$. Let $M$ be an admissible lattice in $V(n)$ determined by the coefficients $\left\{x_{0}, \ldots, x_{n}\right\}$ satisfying the conditions in Lemma 3.1 and $x_{i}>0$ for all $i$.

Let $k$ be a field of characteristic $p$. We denote by $\bar{M}$ the $G_{k}$-module $M \otimes k$, which has a basis $\left\{z_{0} \otimes 1, \ldots, z_{n} \otimes 1\right\}$. Note that, for $j>i$, the $G_{k}$-submodule of $\bar{M}$ generated by $z_{j} \otimes 1$ contains $z_{i} \otimes 1$ if $\frac{x_{j}}{x_{i}}\left(\begin{array}{l}j \\ i\end{array}\right) \not \equiv 0(\bmod p)$ and the $G_{k}$-submodule generated by $z_{i} \otimes 1$ contains $z_{j} \otimes 1$ if $\frac{x_{i}}{x_{j}}\left(\begin{array}{c}n-i \\ n-j\end{array}\right) \not \equiv 0$ $(\bmod p)$. Based on this property, we define the undirected graph $\Gamma(M)$ with vertices $\{0,1, \ldots, n\}$ as follows: for $j>i,\{i, j\}$ is an edge in $\Gamma(M)$ if either $\frac{x_{j}}{x_{i}}\left(\begin{array}{l}j \\ i\end{array}\right) \not \equiv 0(\bmod p)$ or $\frac{x_{i}}{x_{j}}\left(\begin{array}{l}n-i \\ n-j\end{array}\right) \not \equiv 0(\bmod p)$. Note that all weights in $\bar{M}$ have multiplicity 1 . Following the above argument, the weight vectors $z_{i} \otimes 1$ and $z_{j} \otimes 1$ appear in the same indecomposable component of $\bar{M}$ if $\{i, j\}$ is an edge in $\Gamma(M)$. This shows

Lemma. The $G_{k}$-module $\bar{M}$ is indecomposable if and only if the graph $\Gamma(M)$ is connected.

3.3. For each integer $i$, let $v_{p}(i)$ be the $p$-adic valuation of $i$, i.e., the largest nonnegative integer $r$ with $i \equiv 0\left(\bmod p^{r}\right)$.

Proposition. Let $n \geq j>i \geq 0$. Suppose $\{i, j\}$ is not an edge in $\Gamma(M)$. Then

(i) $v_{p}\left(\left(\begin{array}{l}j \\ i\end{array}\right)\right) \geq v_{p}\left(x_{i}\right)-v_{p}\left(x_{j}\right)+1$ and $v_{p}\left(\left(\begin{array}{c}n-i \\ n-j\end{array}\right)\right) \geq v_{p}\left(x_{j}\right)-v_{p}\left(x_{i}\right)+1$,

(ii) if one of $v_{p}\left(\left(\begin{array}{l}j \\ i\end{array}\right)\right)$ and $v_{p}\left(\left(\begin{array}{c}n-i \\ n-j\end{array}\right)\right)$ is 0 , then the other has to be at least 2 .

Proof. Since $\{i, j\}$ is not an edge, then $v_{p}\left(\frac{x_{j}}{x_{i}}\left(\begin{array}{l}j \\ i\end{array}\right)\right) \geq 1$ and $v_{p}\left(\frac{x_{i}}{x_{j}}\left(\begin{array}{l}n-i \\ n-j\end{array}\right)\right) \geq 1$ by the definition. Now (i) follows from the properties of the valuation. To 
show (ii), let, say, $v_{p}\left(\left(\begin{array}{l}j \\ i\end{array}\right)\right)=0$. Then $v_{p}\left(x_{j}\right)-v_{p}\left(x_{i}\right) \geq 1$. Thus $v_{p}\left(\left(\begin{array}{l}n-i \\ n-j\end{array}\right)\right) \geq 2$ following the other inequality in (i).

Corollary. If $\{0, j\}$ is not an edge, then $v_{p}\left(x_{j}\right) \geq 1$ and $v_{p}\left(\left(\begin{array}{l}n \\ j\end{array}\right)\right) \geq 2$.

Proof. Take $i=0$ in the above proposition.

3.4. For a nonnegative integer $n$, we write $n=a_{r} p^{r}+a_{r-1} p^{r-1}+\cdots+a_{0}$ with $0 \leq a_{i}<p$ for $i=0, \ldots, r$. We define $s_{n}=a_{r}+a_{r+1}+\cdots+a_{0}$. It is well known that $v_{p}(n !)=\left(n-s_{n}\right) /(p-1)$ (see [3, p. 263]). Thus, for $0 \leq m \leq n$, we have

$$
v_{p}\left(\left(\begin{array}{c}
n \\
m
\end{array}\right)\right)=v_{p}(n !)-v_{p}(m !)-v_{p}((n-m) !)=\frac{s_{m}+s_{n-m}-s_{n}}{p-1} .
$$

From now on we fix $0 \leq m \leq n$ and write $m=b_{r} p^{r}+\cdots+b_{0}$ in $p$-adic form as well. It follows from the above formula that $v_{p}\left(\left(\begin{array}{c}n \\ m\end{array}\right)\right)=0$ if $a_{i} \geq b_{i}$ for $i=0, \ldots, r$. Otherwise, there is at least one $i$ with $b_{i}>a_{i}$. For each such $i$ if it exists, we define $i^{\prime}$ to be the minimal $i^{\prime}>i$ with $b_{i^{\prime}}<a_{i^{\prime}}$. (Such $i^{\prime}$ always exists.) Let $s\left(i, i^{\prime}\right)=\left\{i, i+1, \ldots, i^{\prime}\right\}$ be the subset of $\{0,1, \ldots, r\}$. Let $T$ be the set of all such $s\left(i, i^{\prime}\right)$. Then $T$ has a partial order under the inclusion relation. We call a maximal member of $T$ a component for the pair $(m, n)$ and denote by $C$ the set of all components for the pair $(m, n)$. For each component $s\left(i, i^{\prime}\right)$, we define $l\left(s\left(i, i^{\prime}\right)\right)=i^{\prime}-i=\left|s\left(i, i^{\prime}\right)\right|-1$, which is called the length of $s\left(i, i^{\prime}\right)$.

Proposition. For each pair $(m, n)$,

(i) different components are disjoint as subsets of $\{0, \ldots, r\}$,

(ii) $v_{p}\left(\left(\begin{array}{c}n \\ m\end{array}\right)\right)=\sum_{s\left(i, i^{\prime}\right) \in C} l\left(s\left(i, i^{\prime}\right)\right)$.

Proof. Let $s\left(i, i^{\prime}\right)$ and $s\left(j, j^{\prime}\right)$ be two components. If $i=j$, then $s\left(i, i^{\prime}\right)=$ $s\left(j, j^{\prime}\right)$ following the definition. We may assume $i<j$. If $s\left(i, i^{\prime}\right) \cap s\left(j, j^{\prime}\right) \neq$ $\varnothing$, then $j<i^{\prime}$. Therefore $j^{\prime} \leq i^{\prime}$ since $a_{i^{\prime}}<b_{i^{\prime}}$. Thus we have $s\left(j, j^{\prime}\right) \subseteq$ $s\left(i, i^{\prime}\right)$ and $s\left(i, i^{\prime}\right)$ is strictly larger than $s\left(j, j^{\prime}\right)$. This contradicts the maximality of a component.

To show (ii), we write $n-m=\sum_{i=0}^{r} c_{i} p^{i}$ with $0 \leq c_{i}<p$. Let $N=$ $\bigcup_{s\left(i, i^{\prime}\right) \in C} s\left(i, i^{\prime}\right)$ and $N^{\prime}=\{0, \ldots, r\} \backslash N$. Now following (i) we can write

$$
n-m=\sum_{k \in N^{\prime}}\left(a_{k}-b_{k}\right) p^{k}+\sum_{s\left(i, i^{\prime}\right) \in C} \sum_{k=i}^{i^{\prime}}\left(a_{k}-b_{k}\right) p^{k}
$$

In the first summation, we have $0 \leq a_{k}-b_{k}<p$. To calculate the second summation, we consider, for each $s\left(\bar{i}, i^{\prime}\right) \in C$,

$$
\sum_{k=i}^{i^{\prime}}\left(a_{k}-b_{k}\right) p^{k}=\left(p+a_{i}-b_{i}\right) p^{i}+\sum_{k=i+1}^{i^{\prime}-1}\left(p+a_{k}-b_{k}-1\right) p^{k}+\left(a_{i^{\prime}}-b_{i^{\prime}}-1\right) p^{i^{\prime}} \text {. }
$$

Here the coefficients of $p^{k}$ are in $\{0, \ldots, p-1\}$. Following the disjointness of the components we get the $p$-adic expansion of $n-m$. Thus we have proved

(i) $c_{k}=a_{k}-b_{k}$ if $k$ is not in any component of $(m, n)$;

(ii) if $s\left(i, i^{\prime}\right)$ is a component of $(m, n)$, then $c_{i}=p+a_{i}-b_{i}, c_{i^{\prime}}=$ $a_{i^{\prime}}-b_{i^{\prime}}-1$, and $c_{k}=p+a_{i}-b_{i}-1$ for $i<k<i^{\prime}$; and

(iii) $\sum_{k=i}^{i^{\prime}}\left(c_{k}+b_{k}-a_{k}\right)=\left(i^{\prime}-i\right)(p-1)$. 
Thus using the above results we have

$$
\begin{aligned}
v_{p}\left(\left(\begin{array}{c}
n \\
m
\end{array}\right)\right) & =\frac{1}{p-1}\left(s_{m}+s_{n-m}-s_{n}\right)=\frac{1}{p-1} \sum_{k=0}^{r}\left(c_{k}+b_{k}-a_{k}\right) \\
& =\frac{1}{p-1}\left(\sum_{k \in N^{\prime}}\left(c_{k}+b_{k}-a_{k}\right)+\sum_{s\left(i, i^{\prime}\right) \in C} \sum_{k=i}^{i^{\prime}}\left(c_{k}+b_{k}-a_{k}\right)\right) \\
& =\frac{1}{p-1} \sum_{s\left(i^{\prime}, i\right) \in C}\left(i^{\prime}-i\right)(p-1)=\sum_{s\left(i, i^{\prime}\right) \in C} l\left(s\left(i, i^{\prime}\right)\right) .
\end{aligned}
$$

3.5. Though the following results are irrelevant to our goal, they have their own interest. The method in the proof of the proposition will be used in the calculation later on.

Proposition. Let $I_{n}=\left\{m \mid 0 \leq m \leq n, v_{p}\left(\left(\begin{array}{l}n \\ m\end{array}\right)\right) \leq 1\right\}$. Then

$$
\left|I_{n}\right|=\prod_{k=0}^{r}\left(a_{k}+1\right)+\sum_{i=0}^{r-1}\left(a_{i+1}\left(p-a_{i}-1\right) \prod_{k \neq i, i+1}\left(a_{k}+1\right)\right) .
$$

Proof. Let $I_{n}^{0}=\left\{m \mid v_{p}\left(\left(\begin{array}{c}n \\ m\end{array}\right)\right)=0\right\}$ and $\left.I_{n}^{1}=\left\{m \mid v_{p}\left(\begin{array}{l}n \\ m\end{array}\right)\right)=1\right\}$. It follows from the calculation in 3.4 that $m \in I_{n}^{0}$ if and only if $a_{k} \geq b_{k}$ for all $k$. Thus we have $\left|I_{n}^{0}\right|=\prod_{k=0}^{r}\left(a_{k}+1\right)$. By Proposition 3.4, $m \in I_{n}^{1}$ if and only if the pair $(m, n)$ has only one component, which must have length 1 . If $s(i, i+1)$ is the component, then $b_{i}>a_{i}, a_{i+1}>b_{i+1}$, and $b_{k} \leq a_{k}$ for $k \neq i, i+1$. Thus $b_{i}$ has $\left(p-a_{i}-1\right)$ choices and $b_{i+1}$ has $a_{i+1}$ choices. This shows that $\left|I_{n}^{1}\right|$ has to be the second summation in the proposition. Now the proposition follows.

Corollary. If $n \geq p$, then $\left|I_{n}\right| \geq p+1$. Furthermore, $\left|I_{n}\right|=p+1$ if and only if $n=p^{r}$ for some $r \geq 1$.

Proof. Since $n \geq p$, there is an $i \geq 0$ such that $a_{i+1}>0$. Using the formula in the proposition, we have

$$
\begin{aligned}
\left|I_{n}\right| & \geq\left(a_{i}+1\right)\left(a_{i+1}+1\right)+a_{i+1}\left(p-a_{i}-1\right) \\
& =a_{i+1} p+a_{i}+1 \geq p+1 .
\end{aligned}
$$

To show the second part, one notes that in the above estimation, the first inequality becomes an equality if and only if $a_{k}=0$ for all $k \neq i, i+1$ and the second inequality becomes an equality if and only if $a_{i}=0$ and $a_{i+1}=1$. Thus the corollary follows.

3.6. We are now ready to prove that the graph $\Gamma(M)$ is connected. We show that there is a path connecting 0 and $m$ by induction on $\left.v_{p}\left(\begin{array}{l}n \\ m\end{array}\right)\right)$. It follows from Corollary 3.3 that $\{0, m\}$ is an edge if $\left.v_{p}\left(\begin{array}{l}n \\ m\end{array}\right)\right) \leq 1$. For $\left.v_{p}\left(\begin{array}{l}n \\ m\end{array}\right)\right) \geq 2$, we show that there is a path connecting 0 and $m$ under the assumption that there a path connecting 0 and $k$ whenever $\left.v_{p}\left(\begin{array}{l}n \\ k\end{array}\right)\right)<v_{p}\left(\left(\begin{array}{l}n \\ m\end{array}\right)\right)$. We use the notations of 3.4 and discuss several cases.

(A) If the pair $(m, n)$ has a component of length 1 , then there is a path connecting 0 and $m$. In fact, let $s(i, i+1)$ be a component of length 1 . Then $a_{i}<b_{i}$ and $a_{i+1}>b_{i+1}$. Take $k=\sum_{t=0}^{r} d_{t} p^{t}$ such that $d_{t}=b_{t}$ for $t \neq i$ and $d_{i}=0$. Then $k<m \leq n$. It follows from Proposition 3.4 that $v_{p}\left(\left(\begin{array}{c}m \\ k\end{array}\right)\right)=0$. Note 
that $\left(\begin{array}{l}n-k \\ n-m\end{array}\right)=\left(\begin{array}{c}n-k \\ m-k\end{array}\right)$ and $m-k=b_{i} p^{i}$. Following the proof of Proposition 3.4, we have $n-k=\sum_{t=0}^{r} e_{t} p^{t}$ with $e_{i}=a_{i}$ and $e_{i+1}=a_{i+1}-b_{i+1}>0$. This shows that the pair $(m-k, n-k)$ has only one component, which has length 1. By Proposition 3.4(ii), $v_{p}\left(\left(\begin{array}{c}n-k \\ m-k\end{array}\right)\right)=1$ and, therefore, $\{k, m\}$ is an edge by Proposition 3.3(ii).

On the other hand, the components of the pair $(k, n)$ are components of $(m, n)$ and $s(i, i+1)$ is not a component for $(k, n)$. Thus $v_{p}\left(\left(\begin{array}{l}n \\ k\end{array}\right)\right)<v_{p}\left(\left(\begin{array}{l}n \\ m\end{array}\right)\right)$ and there is a path in the graph $\Gamma(M)$ connecting 0 and $k$ by the induction hypothesis.

(B) If $(m, n)$ has a component $s\left(i, i^{\prime}\right)$ of length at least 2 with $a_{i^{\prime}-1}>0$, then there is a path connecting 0 and $m$. In this case we take $k=\sum_{t=0}^{r} d_{t} p^{t}$ with $d_{t}=b_{t}$ for $t \neq i^{\prime}-1$ and $d_{i^{\prime}-1}=0$. Then $k<m \leq n$ and $v_{p}\left(\left(\begin{array}{c}m \\ k\end{array}\right)\right)=0$. In addition $m-k=b_{i^{\prime}-1} p^{i^{\prime}-1}\left(b_{i^{\prime}-1} \geq a_{i^{\prime}-1}>0\right)$ and $n-k=\sum_{t=0}^{r} e_{t} p^{t}=$ $\sum_{t=0}^{r} c_{t} p^{t}+b_{i^{\prime}-1} p^{i^{\prime}-1}$. It follows from the proof of Proposition 3.4 that $c_{i^{\prime}}=$ $a_{i^{\prime}}-b_{i^{\prime}}-1$ and $c_{i^{\prime}-1}=p+a_{i^{\prime}-1}-b_{i^{\prime}-1}-1$. Thus $b_{i^{\prime}-1}>e_{i^{\prime}-1}=a_{i^{\prime}-1}-1$ and $e_{i^{\prime}}=a_{i^{\prime}}-b_{i^{\prime}}>0$. This shows that the pair $(m-k, n-k)$ has only one component, which is of length 1 . Thus $v_{p}\left(\left(\begin{array}{c}n-k \\ m-k\end{array}\right)\right)=1$ and $\{k, m\}$ is an edge. On the other hand, the component $s\left(i, i^{\prime}\right)$ for $(m, n)$ changes to the component $s\left(i, i^{\prime}-1\right)$ for $(k, n)$ and all other components of $(m, n)$ are identical to those of $(k, n)$. This shows that $v_{p}\left(\left(\begin{array}{l}n \\ k\end{array}\right)\right) \leq v_{p}\left(\left(\begin{array}{l}n \\ m\end{array}\right)\right)-1$. By induction hypothesis, there is a path in $\Gamma(M)$ connecting 0 and $k$. Thus we have a path connecting 0 and $m$.

(C) If $(m, n)$ has a component $s\left(i, i^{\prime}\right)$ of length at least 2 , then there is a path connecting 0 and $m$. In this case we define

$$
s_{0}=\min \left\{s>i \mid a_{s}<b_{s}\right\}, \quad m_{0}=\min \left\{s>i \mid a_{s}=b_{s}<p-1\right\} .
$$

Here we use the convention $\min \varnothing=+\infty$. Note that $s_{0} \neq m_{0}$ if they exist. There are three possible situations we discuss.

(C1) $s_{0}<m_{0}$ and $s_{0}<i^{\prime}$. In this case, we have $a_{s}=b_{s}=p-1$ for all $i<s<s_{0}$. We take $k=\sum_{t=0}^{r} d_{t} p^{t}$ with $d_{t}=b_{t}$ if $t \neq s_{0}-1$ and $d_{s_{0}-1}=0$. Then $k<m$ and $v_{p}\left(\left(\begin{array}{c}m \\ k\end{array}\right)\right)=0$. The component $s\left(i, i^{\prime}\right)$ of $(m, n)$ splits into (possibly) two components for $(k, n)$. They are proper subsets of $s\left(i, i^{\prime}\right)$. All other components of $(k, n)$ and $(m, n)$ are identical. Thus $v_{p}\left(\left(\begin{array}{l}n \\ k\end{array}\right)\right)<v_{p}\left(\left(\begin{array}{l}n \\ m\end{array}\right)\right)$ and there is a path connecting 0 and $k$. On the other hand, $m-k=b_{s_{0}-1} p^{s_{0}-1}$ and $n-k=\sum_{t=0}^{r} c_{t} p^{t}+b_{s_{0}-1} p^{s_{0}-1}=\sum_{t=0}^{r} e_{t} p^{t}$. Here $c_{s_{0}-1}=p+(p-1)-$ $(p-1)-1=p-1$ if $s_{0}>i+1$ and $c_{s_{0}-1}=p+a_{i}-b_{i}$ if $s_{0}=i+1$. Thus $e_{s_{0}-1}=p-2$ if $s_{0}>i+1$ and $e_{s_{0}-1}=a_{i}$ if $s_{0}=i+1$. In both cases, we have $e_{s_{0}}=c_{s_{0}}+1=p+a_{s_{0}}-b_{s_{0}}>0$ and therefore, the pair $(n-k, m-k)$ has only one component, which is of length 1 . Thus we have $v_{p}\left(\left(\begin{array}{l}n-k \\ n-m\end{array}\right)\right)=1$ and $\{k, m\}$ is an edge.

(C2) $m_{0}<s_{0}$ and $m_{0}<i^{\prime}$. In this case, $a_{s}=b_{s}=p-1$ for $i<s<m_{0}$. Take $k=\sum_{t=0}^{r} d_{t} p^{t}$ with $d_{t}=b_{t}$ for $t \neq m_{0}$ and $d_{m_{0}}=p-1$. Thus $m<k \leq n$ and $v_{p}\left(\left(\begin{array}{l}k \\ m\end{array}\right)\right)=0$. The two pairs $(m, n)$ and $(k, n)$ have exactly the same set of components. Then $v_{p}\left(\left(\begin{array}{l}n \\ k\end{array}\right)\right)=v_{p}\left(\left(\begin{array}{l}n \\ m\end{array}\right)\right)$. But the pair $(k, n)$ satisfies the conditions in $(\mathrm{Cl})$. Thus there is a path connecting 0 and $k$. On the other hand, $k-m=\left(p-1-b_{m_{0}}\right) p^{m_{0}}$ and $n-m=\sum_{t=0}^{r} c_{t} p^{t}$. Following 
the proof of Proposition $3.4 c_{m_{0}}=p+a_{m_{0}}-b_{m_{0}}-1=p-1$. Thus the pair $(k-m, n-m)$ has no component and, therefore, $\left.v_{p}\left(\begin{array}{c}n-m \\ k-m\end{array}\right)\right)=0$. Hence $\{k, m\}$ is an edge in $\Gamma(M)$.

(C3) $m_{0}>i^{\prime}$ and $s_{0}>i^{\prime}$. In this case, $s_{0}>i^{\prime}$ shows that $a_{s}=b_{s}$ for all $i<s<i^{\prime}$. Furthermore, $m_{0}>i^{\prime}$ shows that $a_{s}=b_{s}=p-1$ for $i<s<i^{\prime}$. In particular, $a_{i^{\prime}-1} \neq 0$. This has been proved in (B).

Combining (A) and (C) we have accomplished the induction. Thus we conclude

Theorem. For the Chevalley group $\mathrm{SL}_{2}(k)$, the modules $\bar{M}=M \otimes k$ are indecomposable for all admissible lattices $M$ in $V(n)$ and all nonnegative integers $n$.

Remark. In general, for a semisimple complex Lie algebra $\mathfrak{g}$ and a simple $\mathfrak{g}$ module $V(\lambda)$, it is expected that the resulting modules $M \otimes k$ for the simply connected Chevalley group $G_{k}$ are indecomposable for all admissible lattices $M$ in $V(\lambda)$. Furthermore one may want to distinguish the cohomology groups of line bundles (in generic case) from other modules arising from admissible lattices, e.g., comparing the Loewy lengths.

3.7. One can further ask about the submodule structure of the module $\bar{M}=$ $M \otimes k$ for an admissible lattice $M$ in $V(n)$. If $n<p^{2}$, this is clear since $\bar{M}$ is indecomposable and has only two composition factors. Thus the only two possible structures are $H^{0}(n)$ and $H^{1}\left(s_{\alpha} \cdot n\right)$. But for $n \geq p^{2}$, this is not true anymore.

In our rank 1 situation, a composition factor in a Weyl module has multiplicity 1 . So the submodule structure of $\bar{M}$ could be presented by a directed graph with all composition factors as vertices. The Serre duality shows that the graph for $H^{0}(n)$ is the dual graph (reversing arrows) of the graph for $H^{1}\left(s_{\alpha} \cdot n\right)$. Following the duality we described in $\S 1$, whenever $\Gamma$ is a directed graph of a module $\bar{M}$ for an admissible lattice $M$, the dual graph of $\Gamma$ is the graph for the module $\overline{M^{\prime}}$.

Following the example below, one may have a feeling what the submodule structure of $\bar{M}$ should be. Of course, in the higher rank case when the multiplicity of a composition factor is larger than 1, one may need a better way to describe the submodule structure.

Example. Let $n=4$. There are six different admissible lattices in $V(n)$. If we take $p=2$, the six admissible lattices yield four nonisomorphic indecomposable modules for $\mathrm{SL}_{2}(k)$. The Weyl module has three composition factors with highest weights $0,2,4$ respectively. The graphs of the four modules are given here:

$$
4 \rightarrow 0 \rightarrow 2, \quad 4 \rightarrow 0 \leftarrow 2, \quad 4 \leftarrow 0 \rightarrow 2, \quad 4 \leftarrow 0 \leftarrow 2 .
$$

Here the last one corresponds to $H^{0}(n)$ and the first one to $H^{1}\left(s_{\alpha} \cdot n\right)$, which is the Weyl module. It can be read off from the graphs that $H^{0}(n)$ and $H^{1}\left(s_{\alpha} \cdot n\right)$ have the maximal Loewy length 3. 


\section{REFERENCES}

1. H. H. Andersen, Filtrations of cohomology modules for Chevalley groups, Ann. Sci. École Norm. Sup. (4) 16 (1983), 459-528.

2. S. R. Doty and J. B. Sullivan, On the structure of higher cohomology modules of line bundles on $G / B$, J. Algebra 114 (1988), 286-332.

3. H. Hasse, Number theory, Springer-Verlag, Berlin, Heidelberg, and New York, 1980.

4. J. E. Humphreys, Introduction to Lie algebras and representation theory, Springer-Verlag, New York, Heidelberg, and Berlin, 1972.

5. J. C. Jantzen, Representations of algebraic groups, Academic Press, Orlando, 1987.

6. __, Weyl modules for groups of Lie type, Finite Simple Groups II, Academic Press, London, 1980, pp. 291-300.

7. B. Kostant, Groups over Z, Algebraic Groups and Their Discontinuous Subgroups (A. Borel and G. D. Mostow, eds.), Proc. Sympos. Pure Math., vol. 8, Amer. Math. Soc., Providence, RI, 1966, pp. 90-98.

8. R. Steinberg, Lectures on Chevalley groups, Yale Univ., New Haven, 1968.

9. W. J. Wong, Representation of Chevalley groups in characteristic p, Nagoya Math. J. 45 (1972), 39-78.

Department of Mathematics, University of Washington, Seattle, Washington 98195 